\title{
The Diffusion of Innovations in Social Networks
}

\author{
H. Peyton Young
}

\section{$\underline{1 . \text { Introduction }}$}

New ideas and ways of doing things do not necessarily take hold all at once, but often spread gradually through social networks. In a classic study, Coleman, Katz, and Menzel (1966) showed how doctors' willingness to prescribe the new antibiotic tetracycline diffused through professional contacts. A similar pattern has been documented in the adoption of family planning methods, new agricultural practices, and a variety of other innovations (Rogers and Shoemaker, 1971; Rogers and Kincaid, 1981; Rogers, 1983; Valente, 1995). In the first stage a few innovators adopt, then people in contact with the innovators adopt, then people in contact with those people adopt, and so forth until eventually the innovation spreads throughout the society.

A similar process can be used to describe the diffusion of certain norms of behavior. For example, if people are more likely to jaywalk when they see others in the neighborhood jaywalking, the actions of a few "innovators" may cause jaywalking to become common practice in a given area. This general kind of mechanism has been suggested to explain a variety of social pathologies, including criminality, having children out of wedlock, and dropping out of high school (Crane, 1991; Glaeser, Sacerdote, and Scheinkman, 1996; Akerlof, 1997; Glaeser and Scheinkman, 2000). While such behaviors are not actually innovations, the process by which they spread has similar dynamic properties, namely, the propensity to adopt a behavior increases with the number (or proportion) of some reference group that have adopted it.

A traditional concern in the innovation literature has been to identify the characteristics associated with innovators, that is, people who are the first in their area to adopt. One may then ask how many innovators are needed, and how they need to be dispersed, in order to propel the adoption process forward (Rogers, 1983; Valente, 1995). Implicit in some of this literature is the notion that innovation is essentially a one-way process: once an agent has adopted an innovation, he sticks with it. Yet the same feedback mechanisms that cause innovations to be adopted also cause them to be abandoned: for example, an innovation may die out before the critical threshold or tipping point is reached; indeed, this may happen even after the tipping point is reached due to random events that reverse the adoption 
process. Moreover there appear to be historical cases in which this actually did happen. ${ }^{1}$ Thus, if we want to know how long it takes, in expectation, for a "new" behavior to replace an old one, we must analyze the balance of forces pushing the adoption process forward on the one hand, and those pushing it back on the other.

In this paper we study this problem using an approach pioneered by Blume (1993) and Ellison (1993). ${ }^{2}$ The model treats local feedback effects as a stochastic process: the probability that a given person adopts one of two possible actions in a given time period is assumed to be an increasing function of the number of his or her neighbors who have adopted it. We wish to characterize the waiting time until one of the actions (the "innovation") diffuses in society as a whole. Ellison (1993) showed that, when agents are located around a "ring" and they interact with their near neighbors, the expected waiting time is bounded above independently of the number of agents. In this paper we introduce a structural criterion, called close-knittedness, that can be used to analyze the waiting time problem in much more general situations. Roughly speaking, a group is "close-knit" if its members have a relatively large fraction of their interactions with each other as opposed to outsiders. ${ }^{3}$ We show that when agents have a logistic response function to their neighbors' choices, and they interact in small, close-knit groups, the expected waiting time for diffusion to occur is bounded above independently of the number of agents and independently of the initial state.

\section{The model}

A social network will be represented by a graph $\Gamma$ consisting of a finite set $V$ of vertices, together with a set $\mathrm{E}$ of edges. Each vertex $\mathrm{i}$ represents an agent in the system. A directed edge (i, j) from $\mathrm{i}$ to $\mathrm{j}$ indicates that $\mathrm{j}$ 's actions influence i's actions. The strength of the interaction is given by a nonnegative weight $\mathrm{w}_{\mathrm{ij}}$. The interaction is symmetric if $\mathrm{w}_{\mathrm{ij}}=\mathrm{w}_{\mathrm{ji}}$, in which case we represent the mutual influence between $\mathrm{i}$ and $\mathrm{j}$ by an undirected edge $\{\mathrm{i}, \mathrm{j}\}$. A natural example occurs when the degree of influence is determined by geographic proximity, that is, $w_{i j}$ is inversely related to the distance between $i$ and $j$. In what follows we shall focus on the symmetric case; asymmetric interactions present certain technical complications that require separate treatment.

\footnotetext{
${ }^{1}$ Diamond (1997) discusses instances in which fundamental innovations, such as spears, bone hooks, bows and arrows, pottery, etc., were adopted and later lost by some civilizations, especially those that were small and isolated, as in Polynesia.

${ }^{2}$ For related work on local interaction models see Anderlini and Ianni (1996), Berninghaus and Schwalbe (1996), Blume (1995), Brock and Durlauf (1997), Goyal and Janssen (1996, 1997), Durlauf (1997), and Blume and Durlauf (1999, 2000).

${ }^{3}$ The relationship between network structure and the dynamics of contagion processes has been examined in other settings by Goyal and Janssen (1996), Chwe (2000), and Morris (2000). In these cases (as in ours) the network structure is assumed to be fixed. For models in which the network structure is endogenous see Jackson and Watts (1998), and Mailath, Samuelson, and Shaked (1997).
} 
Assume that each agent has two available choices, $\mathrm{A}$ and $\mathrm{B}$. The state of the process at any given time $\mathrm{t}$ is a vector $\mathbf{x}^{\mathrm{t}} \in\{\mathrm{A}, \mathrm{B}\} \mathrm{V}$, where $\mathrm{x}_{\mathrm{i}}^{\mathrm{t}}$ denotes i's choice at time $\mathrm{t}$. The utility to an agent of choosing $\mathrm{A}$ or $\mathrm{B}$ is assumed to have both an individual and a social component. The individual component of payoff $\mathrm{v}_{\mathrm{i}}\left(\mathrm{x}_{\mathrm{i}}\right)$ results from the agent's idiosyncratic preferences for $\mathrm{A}$ or B irrespective of other agents. The social component of payoff results from the externalities created by the choices of other agents. These externalities may arise from a variety of factors, including demonstration effects, increasing returns, or simply a desire to conform.

A general framework for capturing these effects is to suppose that social payoff takes the form $\sum_{\mathrm{w}_{\mathrm{ij}}} \mathrm{u}\left(\mathrm{x}_{\mathrm{i}}\right.$, $\mathrm{x}_{\mathrm{j}}$ ), where the sum is taken over all edges $\{\mathrm{i}, \mathrm{j}\} \in \mathrm{E}$. The function $\mathrm{u}\left(\mathrm{x}_{\mathrm{i}}, \mathrm{x}_{\mathrm{j}}\right)$ can be interpreted as the payoff function of a two-person game in which each player has the strategies A and B. The weight $\mathrm{w}_{\mathrm{ij}}$ may be interpreted either as the "importance" that i attaches to j's actions, or as the probability that $i$ plays agent $\mathrm{j}$ in a given time period. The total payoff to agent $\mathrm{i}$ in state $\mathbf{x}$ is

$$
\mathrm{U}_{\mathrm{i}}(\mathbf{x})=\sum_{\{\mathrm{i}, \mathrm{j}\} \in \mathrm{E}} \mathrm{w}_{\mathrm{ij}} \mathrm{u}\left(\mathrm{x}_{\mathrm{i}}, \mathrm{x}_{\mathrm{j}}\right)+\mathrm{v}_{\mathrm{i}}\left(\mathrm{x}_{\mathrm{i}}\right) .
$$

To be concrete, imagine that $\mathrm{A}$ is an IBM computer and $\mathrm{B}$ is a MAC. People have different tastes for IBMs versus MACs, which are captured by the functions $v_{i}$. The network externality from interacting with other people with computers is a common effect that is reflected in the function $u$. Its impact on a given individual i depends on the agents with whom i interacts and the importance (or frequency) of the interaction, which is captured by the weight $\mathrm{w}_{\mathrm{ij}}$.

Notice that we may interpret $\mathrm{U}_{\mathrm{i}}(\mathbf{x})$ as the payoff function of an n-person game, where $\mathrm{n}$ is the number of vertices in $\Gamma$ and each player has exactly two strategies, A and B. This is the spatial game on $\Gamma$ induced by the payoff functions $u$ and $v_{1}, 1=i=n .{ }^{4}$ The number and form of the equilibria of the spatial game depend crucially on the topological structure of the graph, as we shall see in a moment. Our principal interest, however, is in the dynamics of the process by which agents adjust their behaviors, both in and out of equilibrium.

To analyze this problem we employ a model due to Blume (1993), which is based on the concept of an Ising model in statistical mechanics (Liggett, 1985). Assume that each individual updates his strategy at random times that are governed by a Poisson arrival process. Without any serious loss of generality we

\footnotetext{
${ }^{4}$ Blume (1993) considered the case where the graph is a finite-dimensional lattice and called the corresponding object a lattice game.
} 
may suppose that each person updates once, on average, per unit time interval. (All of the results go through if instead we assume that individuals have different rates of updating that are bounded above and below by fixed positive numbers.) These updating processes are assumed to be independent among the individuals, so the probability is negligible that more than one person updates at any given time.

When an individual updates, the probability of choosing a given action is assumed to be a logistic function of the payoff difference between the two actions. That is, if $\mathbf{x}_{-\mathrm{i}}$ represents the current choices of the other agents, then the probability that $\mathrm{i}$ chooses $\mathrm{A}$ is

$$
P\left\{i \text { chooses } A \mid \mathbf{x}_{-i}\right\}=e^{\beta\left[U_{i}\left(A, x_{-i}\right)-U_{i}\left(B, x_{-i}\right] /\left[1+e^{\beta\left[U_{i}\left(A, x_{-i}\right)-U_{i}\left(B, x_{-i}\right)\right.}\right]\right.} \text {. }
$$

The probability of choosing $B$ is, of course, one minus this quantity. The parameter $\beta=0$ measures the sensitivity of the agent's response to payoff differences: the larger $\beta$ is, the more likely it is that the agent chooses the action with the higher payoff. The case $\beta=\infty$ corresponds to the strict best response function, in which the unique action with highest utility is chosen with probability one. (If both actions have equal utility each is chosen with probability one-half.) The logit function (2) is standard in the discrete choice literature (McFadden, 1974); it has also been used to model subjects' empirical choice behavior in laboratory situations (Mookherjee and Sopher, 1994, 1997; Camerer and Ho, 1999; McKelvey and Palfrey, 1995).

In what follows it will be useful to write the payoff function $\mathrm{u}(\cdot, \cdot)$ in matrix form as follows:

$\begin{array}{rrr} & \text { A } & \text { B } \\ \text { A } & \text { a, a } & \text { c, d } \\ \text { B } & \text { d, c } & \text { b, b }\end{array}$

In other words, when one's "partner" chooses B, the externality from also choosing B is b, whereas the externality from choosing $\mathrm{A}$ is $\mathrm{c}$, and so forth. We shall assume that there are increasing returns from conformity. This means that matching the partner's choice is better than not matching (b > c and a $>d$. For simplicity we assume that the increasing returns aspect is the same for all pairs of agents who interact. Heterogeneity is captured by differences in the importance weights $\mathrm{w}_{\mathrm{ij}}$, and also by differences in the idiosyncratic preferences for $\mathrm{A}$ and $\mathrm{B}$, that is, by differences in the functions $\mathrm{v}_{\mathrm{i}}(\cdot)$.

The long run behavior of this stochastic process can be analyzed using a potential function. For each state $\mathbf{x}$, let $\mathrm{w}_{\mathrm{AA}}(\mathbf{x})$ be the sum of the weights on all edges $\{i, j\}$ such that $x_{i}=x_{j}=A$.

Similarly, let $\mathrm{w}_{\mathrm{BB}}(\mathbf{x})$ be the sum of the weights on all edges $\{i, j\}$ such that $\mathrm{x}_{\mathrm{i}}=\mathrm{x}_{\mathrm{j}}=\mathrm{B}$. Finally, 
let $\mathrm{v}(\mathbf{x})=\sum \mathrm{v}_{\mathrm{i}}\left(\mathrm{x}_{\mathrm{i}}\right)$ be the sum of the idiosyncratic payoffs in state $\mathbf{x}$. Define the potential of state $\mathbf{x}$ to be

$$
\rho(\mathbf{x})=(\mathrm{a}-\mathrm{d}) \mathrm{w}_{\mathrm{AA}}(\mathbf{x})+(\mathrm{b}-\mathrm{c}) \mathrm{w}_{\mathrm{BB}}(\mathbf{x})+\mathrm{v}(\mathbf{x}) .
$$

We can think of $\mathrm{w}_{\mathrm{AA}}(\mathbf{x})$ and $\mathrm{w}_{\mathrm{BB}}(\mathbf{x})$ as a rough measure of the "areas" of the A-region and the B-region respectively. The potential is therefore a linear combination of the area of the A-region, the area of the B-region, and the idiosyncratic payoffs from choosing A and B. The long-run relative frequency of each state $\mathbf{x}$ is given by the Gibbs distribution

$$
\begin{aligned}
& \mu^{\beta}(\mathbf{x})=e^{\beta \rho(\mathbf{x})} / \sum \mathrm{e}^{\beta \rho(\mathbf{y})} . \\
& y \in \Xi
\end{aligned}
$$

It follows that the log of the likelihood ratio between any two states is just a linear function of their difference in potential. When $\beta$ is sufficiently large (there is little noise in the adjustment process), the long-run distribution will be concentrated almost entirely on the states with high potential. Such states are said to be stochastically stable (Foster and Young, 1990).

\section{Analysis of the potential function}

The potential function has a simple interpretation in terms of the spatial game. For any agent $\mathrm{i}$, the neighborhood of $\mathrm{i}, \mathrm{N}_{\mathrm{i}}=\{\mathrm{j}:\{\mathrm{i}, \mathrm{j}\} \in \mathrm{E}\}$ is the set of agents $\mathrm{j}$ that are linked to $\mathrm{i}$ by an edge. Suppose that the current state is $\mathbf{x}$, and that $\mathrm{i}$ changes strategy from $\mathrm{x}_{\mathrm{i}}$ to $\mathrm{x}_{\mathrm{i}}{ }^{\prime}$. Without loss of generality we can assume that $x_{i}=B$ and $x_{i}{ }^{\prime}=A$. Then the change in i's payoff is

$$
\begin{aligned}
U_{\mathrm{i}}\left(\mathrm{A}, \mathbf{x}_{-\mathrm{i}}\right)-\mathrm{U}_{\mathrm{i}}\left(\mathrm{B}, \mathbf{x}_{-\mathrm{i}}\right)= & (\mathrm{a}-\mathrm{d}) \sum_{\mathrm{j} \in \mathrm{N}_{\mathrm{ij}}: \mathrm{x}_{\mathrm{j}}=\mathrm{A}} \sum_{\mathrm{j}}\left(\mathrm{b} \in \mathrm{N}_{\mathrm{i}}: \mathrm{x}_{\mathrm{j}}=\mathrm{B}\right. \\
& =\rho\left(\mathrm{A}, \mathbf{x}_{-\mathrm{i}}\right)-\rho\left(\mathrm{B}, \mathbf{x}_{-\mathrm{i}}\right) .
\end{aligned}
$$

In other words, the change in i's payoff equals the change in potential. It follows that every pure Nash equilibrium of the spatial game is a local maximum of the potential function; conversely, every local maximum of the potential function corresponds to a pure Nash equilibrium of the game. Typically these equilibria correspond to "patchy" distributions of As and Bs that are locally stable; depending on the geometry of the situation there may be a great many of them. 
It is important to recognize that the states that globally maximize potential do not necessarily maximize social welfare. To illustrate, consider our earlier example in which each person can buy a computer of type A or B. Assume that, in the absence of externalities, everyone would prefer A because it is easier to use. Let us also assume, however, that B networks more efficiently with other computers, and this externality is enough to overcome A's greater ease of use. Suppose, for example, that the payoffs are as follows:

network externality(u) idiosyncratic payoff (v)

A B
A $1,1 \quad 0,0$
$\mathrm{v}(\mathrm{A})=8$
B $0,0 \quad 4,4$
$\mathrm{v}(\mathrm{B})=0$

Let $\mathrm{n}_{\mathrm{A}}(\mathbf{x})$ be the number of agents who choose $\mathrm{A}$ in state $\mathbf{x}$. Then the potential function takes the form:

$$
\rho(\mathbf{x})=\mathrm{w}_{\mathrm{AA}}(\mathbf{x})+4 \mathrm{w}_{\mathrm{BB}}(\mathbf{x})+8 \mathrm{n}_{\mathrm{A}}(\mathbf{x})
$$

whereas the welfare function is

$$
\omega(\mathbf{x})=2 \mathrm{w}_{\mathrm{AA}}(\mathbf{x})+8 \mathrm{w}_{\mathrm{BB}}(\mathbf{x})+8 \mathrm{n}_{\mathrm{A}}(\mathbf{x}) .
$$

It is clear that the all-B state maximizes social welfare, whereas the all-A state maximizes potential. Thus, in the long run, the process results (with high probability) in a state where most people have adopted the less favorable technology. ${ }^{5}$

\section{$\underline{4 .}$ The unravelling problem.}

We turn now to the question of how long it takes for the process to come close to the stochastically stable state, starting from an arbitrary initial state. To illustrate the nature of the problem, consider the

\footnotetext{
5 If everyone is indifferent between $\mathrm{A}$ and $\mathrm{B}$, that is, $\mathrm{v}(\mathrm{x})$ is a constant, the potential function takes the form (a d) $w_{A A}(\mathbf{x})+(b-c) w_{B B}(\mathbf{x})+K$. This is maximized in the all-A state when $a-d>b-c$, and in the all-B state when $b-$ $\mathrm{c}>\mathrm{a}-\mathrm{d}$, that is, the process selects the risk dominant equilibrium. An analogous result holds in many other evolutionary learning models (Kandori, Mailath, and Rob, 1993; Young, 1993; Blume, 1995b; van Damme and Weibull (1999).
} 
following example. The agents are located at the vertices of a square grid, which is embedded on the surface of a torus. Thus everyone has exactly four neighbors, and the weights on all edges are one (see Figure 1). Assume that the idiosyncratic payoffs from choosing A or B are zero; all that matters are the payoffs from externalities, which are given by the following payoff matrix

A B
A $3,3 \quad 0,0$
B $0,0 \quad 2,2$

The corresponding potential function is $\rho(\mathbf{x})=3 \mathrm{w}_{\mathrm{AA}}(\mathbf{x})+2 \mathrm{w}_{\mathrm{BB}}(\mathbf{x})$. Thus the all-A state, A, maximizes potential as well as social welfare.

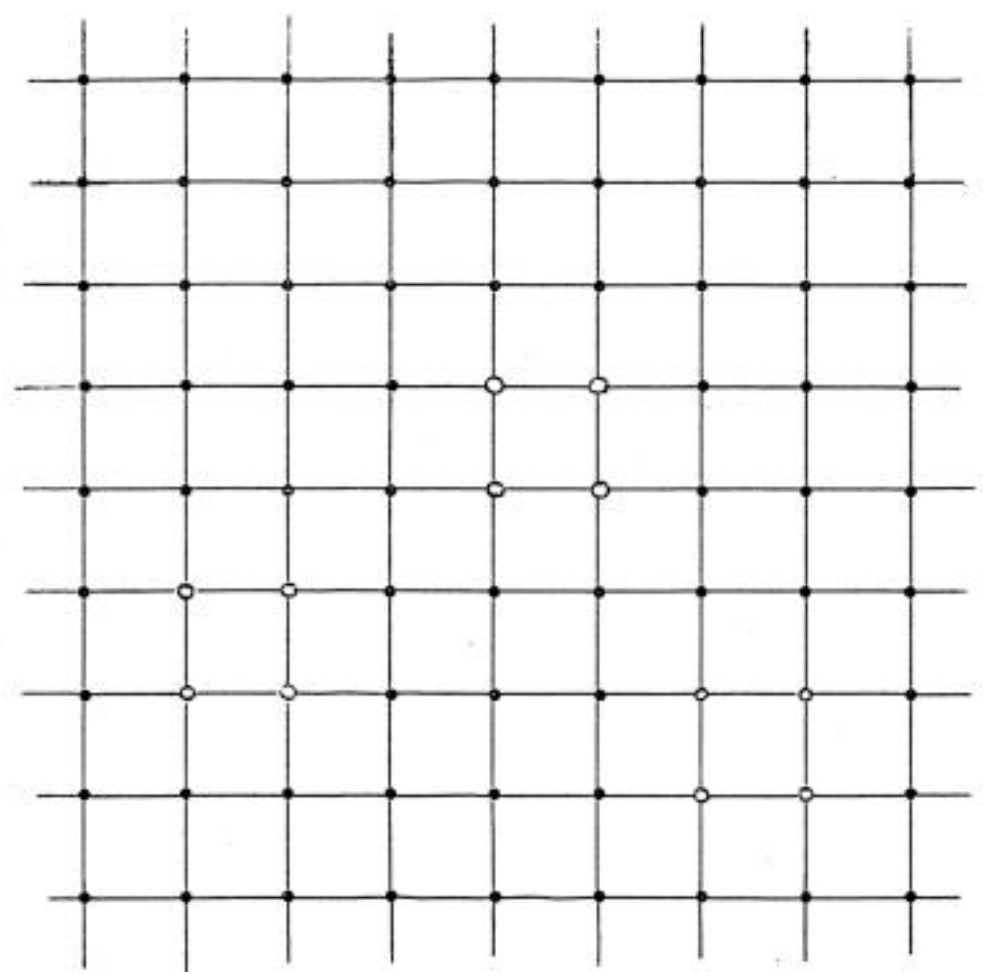

Figure 1. Small enclaves of As (hollow nodes) surrounded by Bs (solid nodes).

Suppose that the process begins in the all-B state, B. Let $\varepsilon=\mathrm{e}^{-\beta}$. In a unit time interval, each agent updates once in expectation. Conditional on updating, an agent surrounded by Bs will switch to A with probability $\mathrm{e}^{0} /\left(\mathrm{e}^{0}+\mathrm{e}^{8}\right) \sim \varepsilon^{8}$. If an agent does switch to $\mathrm{A}$, then in the next time interval each of his four 
neighbors will switch to A with a probability approximately equal to $\varepsilon^{3}$. Eventually, small patches of As will form that are surrounded by Bs. If each such patch forms a rectangle (an "enclave"), then the process is at a Nash equilibrium: no one's payoff increases by switching. But this does not necessarily mean that the process has reached a tipping point from which A spreads rapidly. Indeed, if the Aenclaves are sufficiently small, they are more likely to revert back to B before they expand. This is the "unravelling problem." It can only be overcome once a sufficiently large A-enclave forms, which may take quite some time.

There is a simple geometric criterion which measures the vulnerability of a set to unravelling. Consider any two nonempty subsets of vertices $S$ and $S^{\prime}$, not necessarily disjoint. Define the internal degree of $S^{\prime}$ in $S, d\left(S^{\prime}, S\right)$, to be the number of edges $\{i, j\}$ such that $i \in S^{\prime}$ and $j \in S$. The degree of $i, d_{i}$, is the total number of edges that involve $\mathrm{i}$. The vertex is isolated if $\mathrm{d}_{\mathrm{i}}=0$. Let $\Gamma$ be a graph with no isolated vertices. For every nonempty subset of vertices $S$ in $\Gamma$, we say that $S$ is r-close-knit if the ratio of the internal degree to the total degree is at least $r$ for every nonempty subset of $S$ :

$$
\begin{aligned}
& \min d\left(S^{\prime}, S\right) / S_{i}=r . \\
& S^{\prime} \prod S \quad i \in S^{\prime}
\end{aligned}
$$

This requires, in particular, that every member of $S$ have at least $r$ of its interactions with other members of S. Such a set is said to be r-cohesive (Morris, 2000). In general, however, r-close-knittedness is more demanding than r-cohesiveness. Consider, for example, a 2 x 2 enclave: each member has half of its interactions with other members of the enclave. Then it is $1 / 2$-cohesive, but it is only $1 / 4$-close-knit, because it has four internal edges while the sum of the degrees of its members is 16 . While each vertex taken individually passes muster, the boundary is too large relative to the size of the set.

Not only must the boundary of the whole set be reasonably small for the set to be close-knit, so must every portion of the boundary. In other words, the ratio of internal to total degree must be at least $r$ for every subset, or else the set may begin to unravel at the weakest part of the boundary. This possibility is illustrated in Figure 2: the ratio $\mathrm{d}(\mathrm{S}, \mathrm{S}) / \mathrm{S}_{\mathrm{S}} \mathrm{d}_{\mathrm{i}}$ for the whole group of $\mathrm{As}$ is .4125 , but the ratio $d\left(S^{\prime}, S\right) / S_{S}, d_{i}$ for the dog-leg consisting of four As at the bottom is only .375. For the $2 \times 2$ game in (9) the former is sufficient to prevent unravelling, whereas the latter is not. 


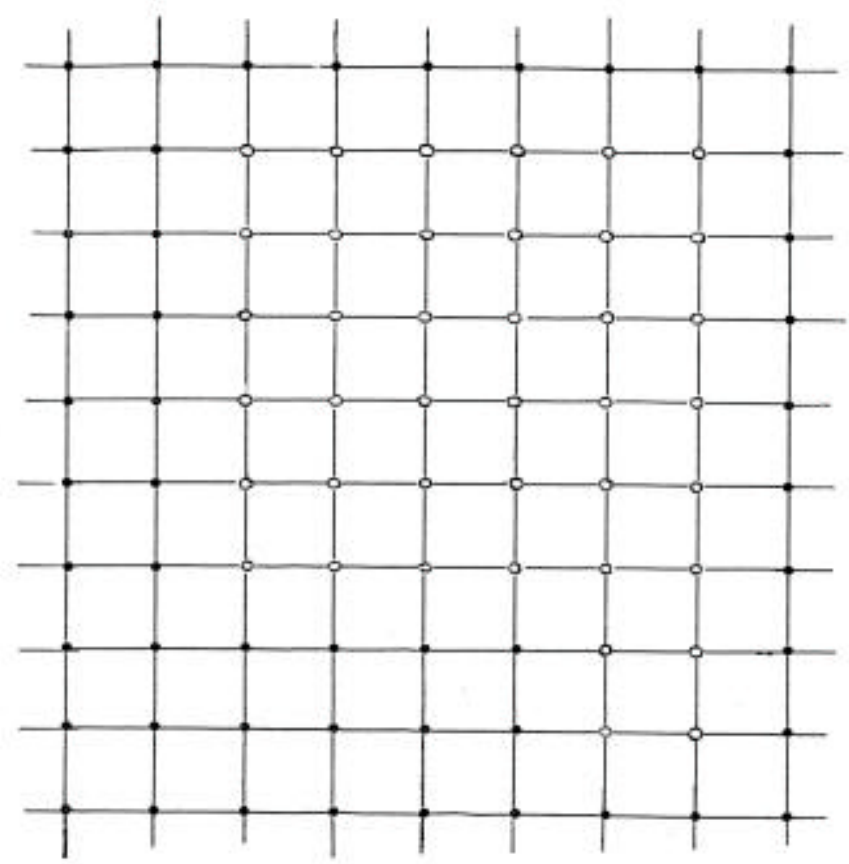

Figure 2. Set of As (hollow nodes) containing a weak dog-leg.

Given a positive real number $\mathrm{r}<1 / 2$ and a positive integer $\mathrm{k}$, we say that a graph $\Gamma$ is $(\mathrm{r}, \mathrm{k})$-close-knit if every person belongs to some group of size at most $\mathrm{k}$ that is at least $\mathrm{r}$-close-knit. A family $\mathrm{F}$ of graphs is close-knit if for every $0<\mathrm{r}<1 / 2$ there exists an integer $\mathrm{k}$ (possibly depending on $\mathrm{r}$ ) such that every graph in the family is $(\mathrm{r}, \mathrm{k})$-close-knit.

As an example, consider the class of all polygons. In a polygon, the degree of every vertex is two.

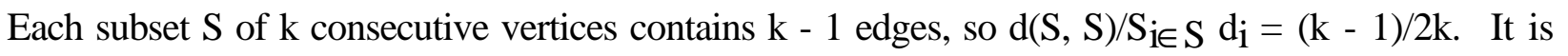
straightforward to check that, in fact, $d\left(S^{\prime}, S\right) / S_{i \in} S^{\prime} d_{i}=(k-1) / 2 k$ for every nonempty subset $S^{\prime}$ of $S$, hence every subset of $k$ consecutive vertices is $(1 / 2-1 / 2 k)$-close-knit. Since every vertex is contained in such a set, the class of polygons is close-knit. For a square lattice embedded on the surface of a torus, it can be verified that every subsquare of side $h$ is $\left(1 / 2-1 / 2 h, h^{2}\right)$-close-knit. It follows that the family of square lattices is close-knit. 


\section{$\underline{\text { 5. A theorem on waiting times }}$}

In this section we show that if a graph comes from a close-knit family, then we can bound the waiting time until the process comes close to a state having maximum potential, and this bound holds uniformly no matter how large the graphs are. Since we need to compare graphs of different sizes, we shall assume henceforth that agents have neutral idiosyncratic preferences $(\mathrm{v}(\mathbf{x})$ is constant) and that all edgeweights are unity. There is no loss of generality in assuming that $\mathrm{v}(\mathbf{x})=0$. The externalities are described by a $2 \times 2$ game $\mathrm{G}$ with payoffs
A B
A a, a c, d
B d, c b, b

Thus the potential function is

$$
\rho(\mathbf{x})=(\mathrm{a}-\mathrm{d}) \mathrm{w}_{\mathrm{AA}}(\mathbf{x})+(\mathrm{b}-\mathrm{c}) \mathrm{w}_{\mathrm{BB}}(\mathbf{x}),
$$

and potential is maximized at the risk dominant equilibrium.

Let $\beta$ be the response parameter, and let $\mathbf{x}^{0}$ be the initial state. Given a small $\delta>0$, let $\mathrm{T}\left(\Gamma, \beta, \mathrm{G}, \delta, \mathbf{x}^{0}\right)$ denote the first time $\tau$ such that, starting from the initial state $\mathbf{x}^{0}$, the probability is at least $1-\delta$ that at least $1-\delta$ of the population is using the risk-dominant equilibrium at $\tau$ and all times thereafter. The $\delta$-inertia of the process defined by $\Gamma, \beta, \mathrm{G}$ is the longest such time over all possible initial states:

$$
\mathrm{T}(\Gamma, \beta, \mathrm{G}, \delta)=\max _{\mathbf{x}^{0}} \mathrm{~T}\left(\Gamma, \beta, \mathrm{G}, \delta, \mathbf{x}^{0}\right) .
$$

The following result generalizes Ellison (1993), who proved that the waiting time is bounded for an essentially one-dimensional process in which the agents are located around a ring. ${ }^{6}$

Theorem. Let $\mathrm{F}$ be a close-knit family of graphs, and let $\mathrm{G}$ be a symmetric $2 \times 2$ game with a risk dominant equilibrium. Given any small $\delta>0$, there exists a $\beta_{\delta}$ such that, for every $\beta=\beta_{\delta}$, the waiting

\footnotetext{
${ }^{6}$ Ellison used a somewhat different stochastic adjustment model in which agents deviate from best reply with a fixed probability $\varepsilon$. In our model they deviate with a probability that depends on the loss in utility.
} 
time $\mathrm{T}(\Gamma, \beta, \mathrm{G}, \delta)$ until the probability is at least $1-\delta$ that at least $1-\delta$ of the population is using the risk dominant equilibrium is uniformly bounded above for all graphs in $\mathrm{F}$.

Proof. Let $\mathrm{F}$ and $\mathrm{G}$ be as in the statement of the theorem. Without loss of generality we can assume that $\mathrm{a}-\mathrm{d}>\mathrm{b}-\mathrm{c}$, that is, $\mathrm{A}$ is the risk dominant equilibrium. Let $\mathrm{r}^{*}=(\mathrm{b}-\mathrm{c}) /((\mathrm{a}-\mathrm{d})+(\mathrm{b}-\mathrm{c}))<1 / 2$ and fix $r \in\left(r^{*}, 1 / 2\right)$. Since $F$ is close-knit, there exists an integer $k$ such that every graph in $F$ is $(r, k)$ close-knit. The parameters $r$ and $\mathrm{k}$ will remain fixed throughout the proof. We are going to show that, given any $\delta \in(0,1)$, there exists $\beta_{\delta}$ such that for each $\beta=\beta_{\delta}$, the $\delta$-inertia of the process is bounded above for all $\Gamma \in \mathrm{F}$.

Choose $\Gamma \in \mathrm{F}$ having vertex set $\mathrm{V}$, and let $\mathrm{S}$ be an $\mathrm{r}$-close-knit subset of size $\mathrm{k}$. The adaptive process on $\mathrm{G}$ will be denoted by $\mathrm{P}^{\Gamma}, \beta$. Now consider the following modification of $\mathrm{P}^{\Gamma}, \beta$ : whenever agents in $\mathrm{S}$ updates, they do so according to the log-linear response process with parameter $\beta$, but the agents in $\overline{\mathrm{S}}$ always choose B. Denote this restricted process by $\mathrm{P} \Gamma, \mathrm{S}, \beta$. States of the restricted process will be denoted by $\mathbf{y}$, and states of the unrestricted process $\mathrm{P} \Gamma, \beta$ will be denoted by $\mathbf{x}$. Let $\Xi_{\mathrm{S}}$ denote the set of restricted states, and $\Xi$ the set of all states.

Let $\mathrm{A}(\mathbf{x})$ denote the number of edges in which both players choose action $\mathrm{A}$ in state $\mathbf{x}$; similarly let $\mathrm{B}(\mathbf{x})$ denote the number of edges in which both players choose action B. By assumption, every agent is indifferent between A and B when there are no externalities, so we can drop the term $\mathrm{v}(\mathbf{x})$ and write the potential function as

$$
\rho(\mathbf{x})=(\mathrm{a}-\mathrm{d}) \mathrm{A}(\mathbf{x})+(\mathrm{b}-\mathrm{c}) \mathrm{B}(\mathbf{x}) .
$$

By (5) we know that the stationary distribution $\mu^{\Gamma, \beta}(\mathbf{x})$ of $\mathrm{P}^{\Gamma, \beta}$ satisfies $\mu \Gamma, \beta(\mathbf{x}) \propto$ e $\beta \rho(\mathbf{x})$ for all $\mathbf{x} \in \Xi$. Similarly it can be shown that the stationary distribution $\mu^{\Gamma, \mathrm{S}, \beta}(\mathbf{y})$ of $\mathrm{P}^{\Gamma, \mathrm{S}, \beta}$ satisfies $\mu^{\Gamma, \mathrm{S}, \beta},(\mathbf{y}) \propto \mathrm{e}^{\beta \rho(\mathbf{y})}$ for all $\mathbf{y} \in \Xi_{S}$.

Let $\mathbf{A}_{S}$ denote the state in $\Xi_{S}$ such that everyone in $S$ chooses action $A$, and everyone in $\bar{S}$ chooses action B. We claim that $\mathbf{A}_{S}$ uniquely maximizes $\rho(\mathbf{y})$ among all restricted states $\mathbf{y}$. To see this, consider any restricted state $\mathbf{y}$ and let $S^{\prime}=\left\{i \in S: y_{i}=B\right\}$. Then

$$
\rho(\mathbf{y})=(\mathrm{a}-\mathrm{d}) \mathrm{d}\left(\mathrm{S}-\mathrm{S}^{\prime}, \mathrm{S}-\mathrm{S}^{\prime}\right)+(\mathrm{b}-\mathrm{c})\left[\mathrm{d}\left(\mathrm{S}^{\prime}, \mathrm{S}^{\prime}\right)+\mathrm{e}\left(\mathrm{S}^{\prime}, \overline{\mathrm{S}}\right)+\mathrm{d}(\overline{\mathrm{S}}, \overline{\mathrm{S}})\right]
$$

and

$$
\rho\left(\mathbf{A}_{\mathrm{S}}\right)=(\mathrm{a}-\mathrm{d}) \mathrm{d}(\mathrm{S}, \mathrm{S})+(\mathrm{b}-\mathrm{c})(\overline{\mathrm{S}}, \overline{\mathrm{S}})
$$

It follows that 


$$
\begin{aligned}
\rho\left(\mathbf{A}_{S}\right)-\rho(\mathbf{y})= & (a-d) d\left(S^{\prime}, S\right)-(b-c)\left[d\left(S^{\prime}, S^{\prime}\right)+d\left(S^{\prime}, \bar{S}\right)\right] \\
& =(a-d) d\left(S^{\prime}, S\right)-(b-c)\left[d\left(S^{\prime}, S^{\prime}\right)+d\left(S^{\prime}, V\right)-d\left(S^{\prime}, S\right)\right]
\end{aligned}
$$

Thus $\rho\left(\mathbf{A}_{\mathbf{S}}\right)-\rho(\mathbf{y})>0$ if and only if

$$
[(a-d)+(b-c)] d\left(S^{\prime}, S\right)>(b-c)\left[d\left(S^{\prime}, V\right)+d\left(S^{\prime}, S^{\prime}\right)\right]
$$

However, the latter holds because by assumption

$$
\begin{aligned}
\mathrm{d}\left(\mathrm{S}^{\prime}, \mathrm{S}\right) / \mathrm{S}_{\mathrm{S}^{\prime}} \mathrm{d}_{\mathrm{i}}=\mathrm{d}\left(\mathrm{S}^{\prime}, \mathrm{S}\right) /\left[\mathrm{d}\left(\mathrm{S}^{\prime}, \mathrm{V}\right)+\mathrm{d}\left(\mathrm{S}^{\prime}, \mathrm{S}^{\prime}\right)\right] \\
>\mathrm{r}^{*}=(\mathrm{b}-\mathrm{c}) /[(\mathrm{a}-\mathrm{d})+(\mathrm{b}-\mathrm{c})] .
\end{aligned}
$$

(Note that $d\left(S^{\prime}, V\right)+d\left(S^{\prime}, S^{\prime}\right)>0$, because by assumption there are no isolated vertices in the graph.) Thus $\mathbf{y}=\mathbf{A}_{\mathbf{S}}$ uniquely maximizes $\rho(\mathbf{y})$ as claimed. It follows that $\mu^{\Gamma, S}, \beta$ puts arbitrarily high probability on the state $\mathbf{A}_{\mathbf{S}}$ whenever $\beta$ is sufficiently large.

Now fix $\delta \in(0,1)$. It follows from the preceding discussion that there exists a finite value $\beta(\Gamma, S, \delta)$ such that $\mu^{\Gamma, S}, \beta\left(\mathbf{A}_{\mathbf{S}}\right)=1-\delta^{2} / 2$ for all $\beta=\beta(\Gamma, \mathbf{S}, \boldsymbol{\delta})$. Fix such a value $\beta$. Consider the restricted

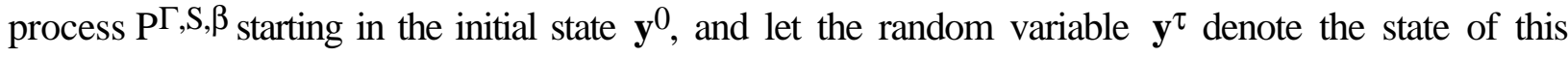
process at time $\tau$. The probability that $\mathbf{y}^{\tau}$ is in any given state $\mathbf{y}$ approaches the long-run probability of $\mathbf{y}, \mu^{\Gamma, S}, \beta(\mathbf{y})$, as $\tau$ goes to infinity. (This follows from the fact that the embedded finite chain is irreducible and aperiodic.) In particular, $\lim _{\tau} \varnothing \in \operatorname{Pr}\left[\mathbf{y}^{\tau}=\mathbf{A}_{S}\right]=\mu^{\Gamma, S}, \beta_{(}\left(\mathbf{A}_{\mathbf{S}}\right)$. Hence there is a finite time $\tau(\Gamma, S, \delta, \beta)$ such that, from any initial state $\mathbf{y}^{0}$,

$$
\forall \beta \varepsilon \beta(\Gamma, S, \delta), \forall \tau=\tau(\Gamma, S, \delta, \beta), \operatorname{Pr}\left[\mathbf{y}^{\tau}=\mathbf{A}_{S}\right]=1-\delta^{2}
$$

Observe now that the continuous process $\mathrm{P} \Gamma, \mathrm{S}, \beta$ depends on $\Gamma$ and $\mathrm{S}$ only through the configuration of internal edges that link vertices of $S$ to other vertices of $S$, and on the configuration of external edges that link vertices of $S$ to vertices outside of $S$. Since $S$ is of size $k$, there is a finite number of internal edges and a finite number of ways in which they can be configured. Since $\mathrm{S}$ is of size k and r-closeknit, there is a finite number of external edges, and a finite number of ways in which they can be configured vis-a-vis vertices outside of $S$. Thus, for a given $r$ and $k$, there is a finite number of distinct processes $\mathrm{P} \Gamma, \mathrm{S}, \beta$ up to isomorphism. In particular, we can find $\beta(\mathrm{r}, \mathrm{k}, \delta)$ and $\tau(\mathrm{r}, \mathrm{k}, \delta, \beta)$ such that, among all graphs $\Gamma$ in $\mathrm{F}$ and all $\mathrm{r}$-close-knit subsets $\mathrm{S}$ with $\mathrm{k}$ vertices, the following holds independently of the initial state: 


$$
\forall \beta=\beta(\mathrm{r}, \mathrm{k}, \delta), \forall \tau=\tau(\mathrm{r}, \mathrm{k}, \delta, \beta), \operatorname{Pr}\left[\mathbf{y}^{\tau}=\mathbf{A}_{\mathrm{S}}\right]=1-\delta^{2}
$$

For the remainder of the discussion, we shall fix $\mathrm{r}, \mathrm{k}$, and $\delta$ as in the theorem. Let us also fix $\beta *=\beta(\mathrm{r}$, $\mathrm{k}, \delta)$ and $\tau *=\tau(\mathrm{r}, \mathrm{k}, \delta, \beta *)$. (In effect, $\beta(\mathrm{r}, \mathrm{k}, \delta)$ is the value $\beta_{\delta}$ claimed in the theorem.)

Let $\Gamma$ be a graph in $F$ with $n$ vertices, and let $S$ be an $r$-close-knit subset in $\Gamma$ of size $\mathrm{k}$. We shall couple the unrestricted process $\Gamma \Gamma, \beta^{*}$ and the restricted process $\mathrm{P} \Gamma, \mathrm{S}, \beta^{*}$ as follows. Create two disjoint isomorphic copies of the graph $\Gamma$, say $\Gamma_{1}$ and $\Gamma_{2}$, where the ith vertex in $\Gamma_{1}$ corresponds to the ith vertex in $\Gamma_{2}$. We will define a single process that mimics $\mathrm{P} \Gamma, \beta^{*}$ on $\Gamma_{1}$, and mimics $\mathrm{P} \Gamma, \mathrm{S}, \beta^{*}$ on $\Gamma_{2}$. For each state $\mathbf{x}$ of the unrestricted process $\mathrm{P}^{\Gamma}, \beta^{*}$, let $\mathrm{q}_{\mathrm{i}}(\mathrm{A} \mid \mathbf{x})$ denote the probability that $\mathrm{i}$ chooses $\mathrm{A}$ when $\mathrm{i}$ updates, given that the current state is $\mathbf{x}$. Similarly, for each state $\mathbf{y}$ of the restricted process $\mathrm{P} \Gamma, \mathrm{S}, \beta^{*}$, let $\mathrm{q}_{\mathrm{i}}^{\prime}(\mathrm{A} \mid \mathbf{y})$ denote the probability that $\mathrm{i}$ chooses $\mathrm{A}$ when $\mathrm{i}$ updates, given that the current state is $\mathbf{y}$. Note that $\mathrm{q}_{i}^{\prime}(\mathrm{A} \mid \mathbf{y})=0$ for all $\mathrm{i} \in \overline{\mathrm{S}}$.

The coupled process operates as follows. The state at time $\tau$ is a pair $\left(\mathbf{x}^{\tau}, \mathbf{y}^{\tau}\right)$ where $\mathrm{x}_{\mathrm{i}}$ is the choice (A or B) at the ith vertex in $\Gamma_{1}$, and $\mathrm{y}_{\mathrm{i}}$ is the choice (A or B) at the ith vertex in $\Gamma_{2}$. Each matched pair of vertices in the two graphs is governed by a single Poisson process with unit expectation, and these processes are independent among the $n$ matched pairs. Thus whenever the ith agent in $\Gamma_{1}$ updates the ith agent in $\Gamma_{2}$ updates, and vice versa. Let $\Theta$ be a random variable that is distributed uniformly on the interval $[0,1]$. Suppose that the ith pair of individuals updates at time $\tau$. Draw a value of $\Theta$ at random, and denote it by $\theta$. The ith individual in $\Gamma_{1}$ chooses $A$ if $\theta=q_{i}\left(A \mid x^{\tau}\right)$ and chooses $B$ if $\theta>q_{i}\left(A \mid x^{\tau}\right)$. Similarly, the ith individual in $\Gamma_{2}$ chooses $A$ if $\theta=q_{i}^{\prime}\left(A \mid y^{\tau}\right)$ and chooses $B$ if $\theta>q_{i}^{\prime}\left(A \mid y^{\tau}\right)$.

For every two states $\mathbf{x}$ and $\mathbf{y}$ on $\Gamma_{1}$ and $\Gamma_{2}$ respectively, write $\mathbf{x} \varepsilon_{\mathrm{A}} \mathbf{y}$ if $y_{\mathrm{i}}=\mathrm{A}$ implies $\quad \mathrm{x}_{\mathrm{i}}=\mathrm{A}$ for all i. In other words, if $A$ appears at the ith vertex in $\mathbf{y}$ then $A$ appears at the ith vertex in $\mathbf{x}$. It is evident that $\mathbf{x}={ }_{\mathrm{A}} \mathbf{y}$ implies $\mathrm{q}_{\mathrm{i}}(\mathrm{A} \mid \mathbf{x})=\mathrm{q}_{\mathrm{i}}^{\prime}(\mathrm{A} \mid \mathbf{y})$ for all $\mathrm{i}$. By construction of the process, if $\mathrm{i}$ chooses $\mathrm{A}$ in $\mathbf{y}^{\tau}$ then necessarily i chooses $\mathrm{A}$ in $\mathbf{x}^{\tau}$. Hence if $\mathbf{x}^{\tau}={ }_{\mathrm{A}} \mathbf{y}^{\tau}$ at some time $\tau$, then $\mathbf{x}^{\tau^{\prime}}={ }_{\mathrm{A}} \mathbf{y}^{\tau^{\prime}}$ at all subsequent times $\tau^{\prime}=\tau$.

Now let the coupled process begin in the initial state $\mathbf{x}^{0}$ on $\Gamma_{1}$ and $\mathbf{y}^{0}$ on $\Gamma_{2}$, where $x_{i}^{0}=y_{i}^{0}$ for all $i$ $\mathrm{S}$, and $\mathrm{y}_{\mathrm{i}}{ }_{\mathrm{i}} \mathrm{B}$ for all i $\quad \overline{\mathrm{S}}$. Obviously we have $\mathbf{x}^{0}={ }_{\mathrm{A}} \mathbf{y}^{0}$ initially, hence we have $\mathbf{x}^{\tau}={ }_{\mathrm{A}} \mathbf{y}^{\tau}$ for all $\tau=$ 0 . From (17) and the choice of $\tau^{*}$ we know that

$$
\forall \tau=\tau^{*}, \quad \operatorname{Pr}\left[\mathbf{y}_{\mathrm{i}} \tau=\text { A for all } \mathrm{i} \in \mathrm{S}\right]=1-\delta^{2},
$$

hence 


$$
\forall \tau=\tau^{*}, \quad \operatorname{Pr}\left[\mathbf{x}_{\mathrm{i}}^{\tau}=\mathrm{A} \text { for all } \mathrm{i} \in \mathrm{S}\right]=1-\delta^{2}
$$

This holds for every r-close-knit set $\mathrm{S}$ in $\Gamma$. Since every vertex i is, by hypothesis, contained in such an $S$, it follows that

$$
\forall \tau=\tau *, \forall \mathrm{i} \quad \operatorname{Pr}\left[\mathbf{x}_{\mathrm{i}}=\mathrm{A}\right]=1-\delta^{2}
$$

Letting $\alpha^{\tau}$ be the proportion of individuals in $\Gamma_{1}$ playing action $A$ at time $\tau$, it follows that

$$
\forall \tau=\tau *, \quad \mathrm{E}\left[\alpha^{\tau}\right]=1-\delta^{2}
$$

We claim that this implies

$$
\forall \tau=\tau *, \operatorname{Pr}\left[\alpha^{\tau}=1-\delta\right]=1-\delta
$$

If this were false, the probability would be greater than $\delta$ that more than $\delta$ of the individuals at time $\tau$ were playing B. But this would imply that $E\left[\alpha^{\tau}\right]<1-\delta^{2}$, contradicting (22). Thus (23) holds for all graphs $\Gamma$ in the family $\mathrm{F}$, which concludes the proof of the theorem. 


\section{References}

Anderlini, Luc, and Antonella Ianni. 1996. "Path Dependence and Learning from Neighbors." Games and Economic Behavior 13:141-77.

Akerlof, George A. 1997. "Social Distance and Social Decisions." Econometrica 65:1005-27.

Berninghaus, S., and Schwalbe, U. (1996). "Conventions, Local Interaction, and Automata Networks," Journal of Evolutionary Economics, 6, 297-312.

Blume, Larry. 1993. "The Statistical Mechanics of Strategic Interaction." Games and Economic Behavior 4: 387-424.

1995a. "The Statistical Mechanics of Best-Response Strategy Revision." Games and Economic Behavior 11: 111-45.

---------------- (1995b). "How Noise Matters." Mimeo, Department of Economics, Cornell University.

Blume, Larry, and Steven N. Durlauf, 1999. "Equilibrium Concepts for Models with Social Interactions," Mimeo, Cornell University.

--------------. 2000. "The Interactions Based Approach to Socioeconomic Behavior," Forthcoming in Social Dynamics, edited by Steven N. Durlauf and H. Peyton Young. The Brookings Institution, Washington, D. C.

Brock, William A., and Steven N. Durlauf. 1997. "Discrete Choice with Social Interactions." Mimeo, University of Wisconsin at Madison, and forthcoming in the Review of Economic Studies.

Camerer, Colin, and Teck-Hua Ho. 1999. "Experience-Weighted Attraction Learning in Normal Form Games." Econometrica 67: 827-874.

Chwe, Michael, 2000. "Communication and Coordination in Social Networks," Review of Economic Studies, 67, 1- 16. 
Coleman, James S., Elihu Katz, and Herbert Menzel. 1966. Medical Innovation: A Diffusion Study. New York: Bobbs Merrill.

Crane, Jonathan. 1991. "The Epidemic Theory of Ghettos and Neighborhood Effects on Dropping Out and Teenage Childbearing." American Journal of Sociology 96: 1226-59.

Diamond, Jared, 1997. Guns, Germs, and Steel. New York: Norton.

Durlauf, Steven N. 1997. "Statistical Mechanical Approaches to Socioeconomic Behavior." In The Economy as a Complex Evolving System. vol. 2, edited by W. Brian Arthur, Steven N. Durlauf, and David Lane. Redwood City, Calif.: Addison-Wesley.

Ellison, Glenn. 1993. "Learning, Social Interaction, and Coordination." Econometrica 61: 1047-71. 2000. "Basins of Attraction, Long-Run Stochastic Stability, and the Speed of Step-by Step Evolution." Review of Economic Studies, 67, 17-45 .

Foster, Dean P. and H. Peyton Young, "Stochastic Evolutionary Game Dynamics." Theoretical Population Biology 38: 219-32.

Glaeser, Edward L., Bruce Sacerdote, and Jose A. Scheinkman. 1996. "Crime and Social Interactions." Quarterly Journal of Economics 11: 507-48.

Glaeser, Edward L., and Jose A. Scheinkman. 1998. "Measuring Social Interactions." Forthcoming in Social Dynamics, edited by Steven N. Durlauf and H. Peyton Young. The Brookings Institution, Washington, D. C.

Goyal, Sanjeev, and Maarten Janssen. 1996. "Interaction Structure and Social Change." Journal of Institutional and Theoretical Economics, 152, 472-495.

1997. "Non-Exclusive Conventions and Social Coordination." Journal of Economic Theory, 77, 34-57.

Jackson, Matthew O., and Alison Watts. 1998. "The Evolution of Social and Economic Networks." Mimeo, California Institute of Technology and Vanderbilt University. 
Kandori, Michihiro, George Mailath, and Rafael Rob. 1993. "Learning, Mutation, and Long-Run Equilibria in Games." Econometrica 61: 29-56.

Liggett, Thomas. 1985. Interacting Particle Systems. New York; Springer Verlag.

Mailath, George, Samuelson, Larry, and Avner Shaked, 1997. "Endogenous Interactions." in U. Pagano and A. Nicita, eds., The Evolution of Economic Diversity. London: Routledge.

Morris, Stephen. 2000. "Contagion." Review of Economic Studies 67, 57-78.

McKelvey, Richard, and Thomas Palfrey. 1995. "Quantal Response Equilibria for Normal Form Games." Games and Economic Behavior 10: 6-38.

Mookherjee, Dilip, and Barry Sopher. 1994. "Learning Behavior in an Experimental Matching Pennies Game." Games and Economic Behavior 7: 62-91.

Games and Economic Behavior 19: 97-132.

Rogers, Everett M. 1983. Diffusion of Innovations. 3rd edition. New York: Free Press.

Rogers, Everett M, and F. F. Shoemaker. 1971. Communication of Innovations: A Cross-Cultural Approach. New York: Free Press.

Rogers, Everett M., and D. L. Kincaid. 1981. Communication Networks: A New Paradigm for Research. New York: Free Press.

Valente, Thomas W. 1995. Network Models of the Diffusion of Innovations. Creskill, NJ: Hampton Press.

1996. "Social Network Thresholds in the Diffusion of Information." Social Networks

18: 69-89.

van Damme, Eric, and Joergen Weibull. 1999. "Evolution and Refinement with Endogenous Mistake Probabilities." Mimeo, Stockholm School of Economics and Center for Economic Research, Tilburg University. 
Young, H. Peyton, 1993a. "The Evolution of Conventions." Econometrica 61: 57-94.

1998. Individual Strategy and Social Structure: An Evolutionary Theory of Institutions. Princeton: Princeton University Press. 


\title{
The Diffusion of Innovations in Social Networks
}

\author{
H. Peyton Young \\ Department of Economics \\ Johns Hopkins University \\ Baltimore, MD 21218
}

This version: May, 2000

This research was supported by National Science Foundation Grant SES 9818975. The paper benefited from constructive comments by Dean Foster and Stephen Morris. 


\begin{abstract}
We consider processes in which new technologies and forms of behavior are transmitted through social or geographic networks. Agents adopt behaviors based on a combination of their inherent payoff and their local popularity (the number of neighbors who have adopted them) subject to some random error. We characterize the long-run dynamics of such processes in terms of the geometry of the network, but without placing a priori restrictions on the network structure. When agents interact in sufficiently small, close-knit groups, the expected waiting time until almost everyone is playing the stochastically stable equilibrium is bounded above independently of the number of agents and independently of the initial state.
\end{abstract}

